\title{
Network Optimization Based Lane Line Classification Study
}

\author{
Hai Wang1, Pengyi Deng ${ }^{1,2,3,}$, Shulei Sun ${ }^{1,2,3}$, Guoying Tian ${ }^{1,2,3}$, Shuguang $\mathrm{Li}^{4}$, Ningyan Luo ${ }^{1}$, Chuilei Kong', Jinbo \\ Zhong1, Yiqiang Peng1,2,3, Junjun Liu \\ 1 the School of Automobile and transportation, Xihua University, Chengdu, China; \\ 2 The Key Laboratory of Automobile Measurement, Control and Safety of Sichuan Province, China \\ 3 The Engineering Research Center of New Energy Vehicle Intelligent Control and Simulation Test Technol- \\ ogy in Sichuan Province \\ 4 the School of Automation Engineering, University of Electronic Science and Technology of China \\ * Correspondence: 961499493@qq.com;
}

\begin{abstract}
Efficient quality evaluation provides support for the timely and good maintenance of the lane line marking. This paper searches and optimizes the back propagation(BP) network model which referred to the analytic hierarchy process(AHP) model structure, as well as the number of nodes in the middle layer network. Based on this, a comprehensive evaluation method of multidimensional lane line quality such as shape, color and contrast is established. The experimental results show that the parameters of the model are more simplified, and the scoring and classification results of lane lines are more accurate.
\end{abstract}

Keywords: lane line, comprehensive evaluation, BP, parameter search, maintenance

\section{Introduction}

As the guarantee of traffic order and social operation, lane line is an important facility of the transportation system, and effects on traffic flow constraints and guidance. Therefore its maintenance plays an vital supporting role in the entire road life cycle[1]. In addition, lane marking, with its color nature, is one of the recognition information for routing and behavior planning in current self-driving system [2-3]. In Ref.[1][4-5] lane line is gradually wear during using, and timely maintenance is of great significance for traffic efficiency. The research focus on realizing fast, efficient and automated road quality monitoring has practical value.

The road maintenance research work is mainly focused on the quality monitoring of pavement (asphalt and other materials), including the identification of potholes, cracks and other defects. The equipment used in these works includes acceleration sensors [6-7] and image sensors [8], and the methods of classification in this routine include clustering[8], support vector machine[9], random forest[10]. The recognition of lane line defects includes two routes: laser point cloud [11-12] and image[14-15]. Laser point cloud can effectively identify lane line shape features[11], but marking's color information cannot be extracted and processed well in this way. In terms of using images for research, literature [14] proposed a systematic method for automatically measuring road marking wear (erosion) using digital image analysis technology, which provided basic research for pixellevel lane line positioning. The technology digitizes the road surface and then employed methods such as color space conversion, threshold segmentation, image enhancement. Lin Kuoliang et al. [15] processed the original image of the lane line based on classic machine vision algorithms to obtain the edge information, and further conducted specific algorithms to quantify the edge curve of the lane line. The team enrolled 5 experts to score data set, with a range of $0-10$, divided into 5 grades, and further used a threshold to divide the data samples into two categories(qualified and un-qualified), to verify the evaluation system. The verification confirms the effectiveness of the system. The system achieved a prediction accuracy of $76.67 \%$ under the $60 \%$ threshold, and pre-cision reached $100 \%$, but the system recall rate was only $50 \%$, which means that the system can accurately identify 
good markings, but in poor performance for unqualified lane lines. Decreasing the threshold value of $50 \%$ will achieve prediction accuracy of $86.67 \%$, precision of $85.71 \%$, and recall rate of $85.71 \%$.

In summary, the existing research mainly focuses on the monitoring of road defects, which mainly focus on the potholes and cracks. The research work related to lane line quality monitoring is mainly on the lane line shape and completeness recognition. The monitoring tools mainly include lidar and camera devices.

Based on the latest lane line construction \& acceptance standards [16-18] and maintenance standards[5], this work expanding the scope of lane line quality monitoring, e.g. color defects, including color deviation, dirt, etc., and giving a more comprehensive and comprehensive automatic monitoring method are aspects that can be further studied at present. In response to this problem, this paper extracted multi-dimensional feature data based on machine vision methods, and proposed a comprehensive quality evaluation method based on network optimization of lane line multi-index quantification.

The appearance quality of lane lines is embodied in various properties such as shape and color. For obtaining object pixel-level feature data, traditional machine vision methods are more stable and accurate[19-20]. In order to establish an evaluation method that can comprehensively examine various quality aspects on this basis, and the method should have a reasonable emphasis on each quality aspect. As a result, it is important to select an appropriate evaluation model .

At present, the research on such multi-index evaluation problems mainly employed AHP [21-22] , fuzzy comprehensive evaluation method, expandable comprehensive evaluation method, TOPSIS, entropy weight method, data envelopment analysis (DEA), BP neural network. For comprehensive quality evaluation, AHP[23-26] and BP[27-28] neural network have been widely applied. The AHP method is easy to use in problem analysis, and the BP network has strong adaptability and good fitting performance in parameter fitting. The AHP-BP method, however, provides a way for the establishment of an evaluation model for complex issues[29]. Considering the advantages of two methods respectively, this article comprehensively study the AHP model and BP network model, construct an AHP-BP net. It conduct parameter search on the transfer function and the number of nodes in the middle layer, and get the best model setting. In this paper, we compare the performance of AHP-BP model, the Ref.[15] on lane line quality evaluation, and validate the efficiency of AHP-BP. The main contributions of this paper can be summarized as follows:

1、 The model adopted in this paper expands the characteristic dimensions of the existing lane line quality recognition classification, so that the lane line quality evaluation meets the actual standards.

2、 Using parameter search method to construct the AHP-BP network has better performance than AHP model, including an optimal performance network and a lightweight network.;

3、 Optimized the AHP-BP method(BP-Mode-1- tansig-purelin-81) performed excellent classification in given database. Compared with the AHP model, Accuracy and recall have a large improvement .Lightweight model(BP-Mode-1- poslin-poslin-14) has $82 \%$ fewer parameters than the optimal network (BP-Mode-1- tansig-purelin-81), but there is only a small decrease compared to the optimal model.

4. The AHP model and the AHP-BP Network model obtained in this paper have greatly improved compared with the method similar literature reported Lane line quality classification.

The remaining content of this article is mainly divided into two parts. The first part is the elaboration of the research methods, mainly including the preprocessing methods for obtaining various feature data of lane lines, the method of constructing the AHP model, and the last about the construction method of the AHP-BP model. The second part is the 
demonstration and discussion part of the experimental results. This part will give the evaluation results based on the method, and prove the effectiveness through comparison.

\section{Materials and Methods}

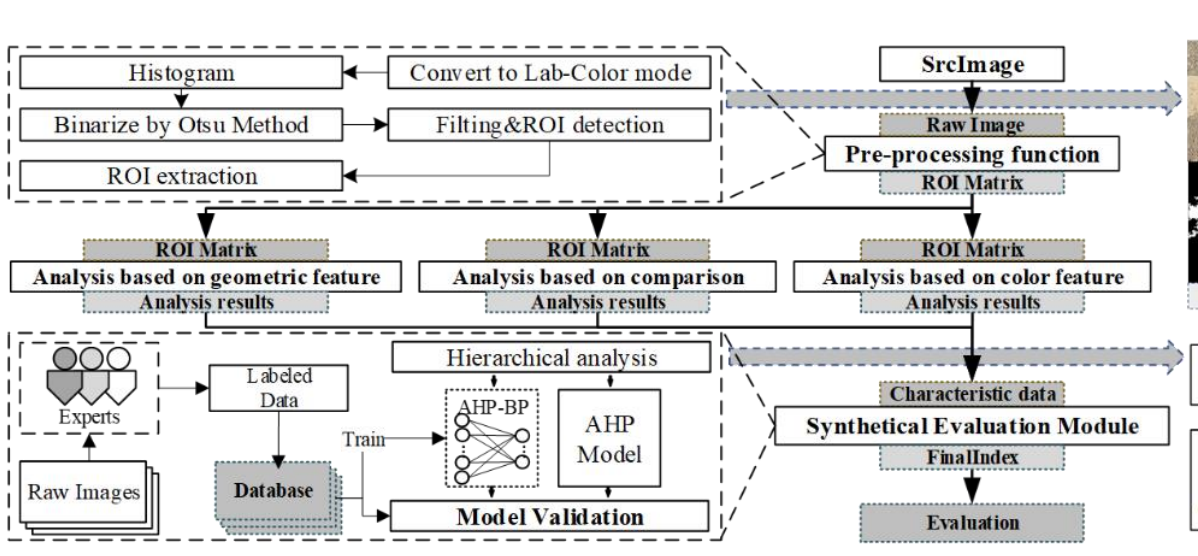

(A)
(B-1)

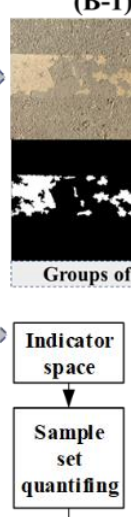

Groups of image in different quality after preprocessing.

(B)

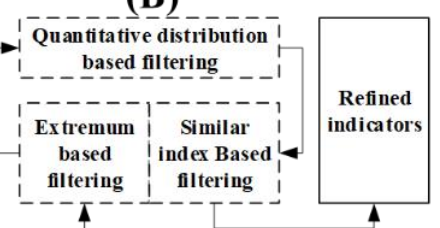

(C)

Fig. 1 (A)Research Plan;(B) pre-processing Performance; (C)Indicators Establishment

\subsection{Image Pre-processing}

Image pre-processing is used to process the original lane marking image for accurate lane line data. In order to obtain the data, the channel data separation[30], Otsu method[31], median filter [32] and connected graph search method are used. Based on channel data , through histogram analysis, it has found that in regard to the yellow lane line, the road surface and the lane line in the Lab$\mathrm{b}$ channel of the image show a strong contrast property, which is conducive for the determination of segmentation threshold. The Otsu method is used to segment adaptively the lane line pixel area. Furthermore, the median filter method is used to reduce the impact of noise after image segmentation. Finally, the ROI area of the lane line can be extracted by the connected image detection. The Image pre-processing has implemented by MATLAB, and its performance under different lane line quality conditions has shown in Fig. 1 (B). It can be seen from Fig. 1 (B-2) (B-3) that the algorithm has a relatively reliable capability.

\subsection{Analytic Hierarchy Process Model}

The analytic hierarchy process model was established through the following steps: 1) construct a hierarchical structure according to the cartain problem; 2) obtain and test the evaluation matrix; 3) calculate the model weight. The construction of the AHP evaluation model needs to analyze the specific problem and its interaction of internal elements. According to the AHP analysis method[21], the model in this article adopted a three-layer structure, namely the target layer, the criterion layer and the basic index layer. The target layer is the total score of the lane line marking; and the criterion layer is the closely quality-related aspects that make up the total score; the basic index is the lower-level influencing indicators of the criterions, which will determine the score of the criterion layer, and further affect the total score. The initial value of the indicators' definition and value were determined by the actual lane line quality. According to the existing lane line construction acceptance standards[16-18] and related maintenance standards[5], the main quality guidelines of lane line in shape and color have shown as A) D).

A. Marking lines should have good visibility, uniform color, neat edge, smooth line[17].

B. The thickness of the marking coating should be uniform, without obvious blistering, wrinkles, spots, cracks, stickiness, peeling, blooming and other defects[17].

C. The surface of the reflective line should be evenly sprinkled with glass beads, and its performance and particle size distribution meet the requirements of GB/T 24722[17]. 
D. Road markings should always be kept in good technical condition, with smooth line shape, clear visual recognition, clean surface, color, size (length, width, spacing, thickness), and reflective performance in compliance with the specification requirements [5].

Due to the inherent nature of machine vision[14], it taken the above A), B), D) as core, and summarized six criteria 1) 6) closely related to the overall appearance quality.

1) Color identity: it reflects whether the color of the marking is consistent or not, whether it is stained or not;

2) Color compliance: it reflect whether the overall color of the marking meets the standard;

3) Width compliance: it reflect whether the lane line width meets the present standard;

4) Shape conformity: it reflects whether there are defects or breaks inside and outside the marking;

5) Edge continuity: it reflects whether the edge of the marking line is continuous and whether there is jagged defect;

6) Contrast with the background: it reflects the visual recognition performance of the marking under a certain road surface.

After determining the criterion-level indicators, it is necessary to obtain the corresponding indicators based on the actual condition. According to the literature [21], the establishment of evaluation system should be able to in line with: the index should be simple; the index should be independent, without causality to peer index; the index should be representative, reflecting the characteristics of a certain aspect of the object ; the indicators are feasible, match with the actual level, and operate available. The specific basic indicators have established according to the process shown in Fig. 1 (C). The following takes the color quality as an example. We have extracted the 9 color channels in the three color modes, including RGB, Lab, HSV. Based on the channel data of different modes, the average deviation and standard deviation based on the value required, and the standard deviation based on the channel mean, have calculated. Among the three calculation methods, the former two were used to measure color conformity with the standard's, and the latter was used to quantify color consistency. After quantification, in terms of color, there were a total of 27 indicators, which have successively passed through three index screening mechanisms based on extreme value Fig. 2, quantitative distribution Fig. 3, and similarity Fig. 4. The quantitative distribution filter was based on formula (1) to determine the distribution coefficient, and according to the sample set quality distribution Fig. 3(a \& b Index 18), the abnormal distribution indictors $(4,5,15,17)$ (shown in Fig. 3) were filtered out, and the similarity screening was based on formula (2) (3) (4), through these method the similarity index between indicators has taken, and group them with $95 \%$ (the similarity threshold) as $(1,2,3),(4,5,7,9),(6),(8),(10,11,12),(13)$ (Fig. 4), then selected the highest quantitative distribution index as the representative of each group, finally through these steps 6 simplified indexes could be get. Similarly, from the geometric characteristics and contrast properties of lane lines with road surface, it obtained the basic indicators of the other four sets of criterion layers, as shown in TABLE A1.

$$
I=\sum_{i=1}^{n}\left(\frac{x_{i}-\min }{\max -\min } * 2-1\right)
$$

Where $\min 、 \max$ represents the minimum and maximum values in the quantized future. The function of $\frac{x_{i}-\min }{\max -\min } * 2-1$ was used to normalize the data into $-1 \sim 1$. And Fig. $3(\mathrm{~b})$ is obtained by this formula. 


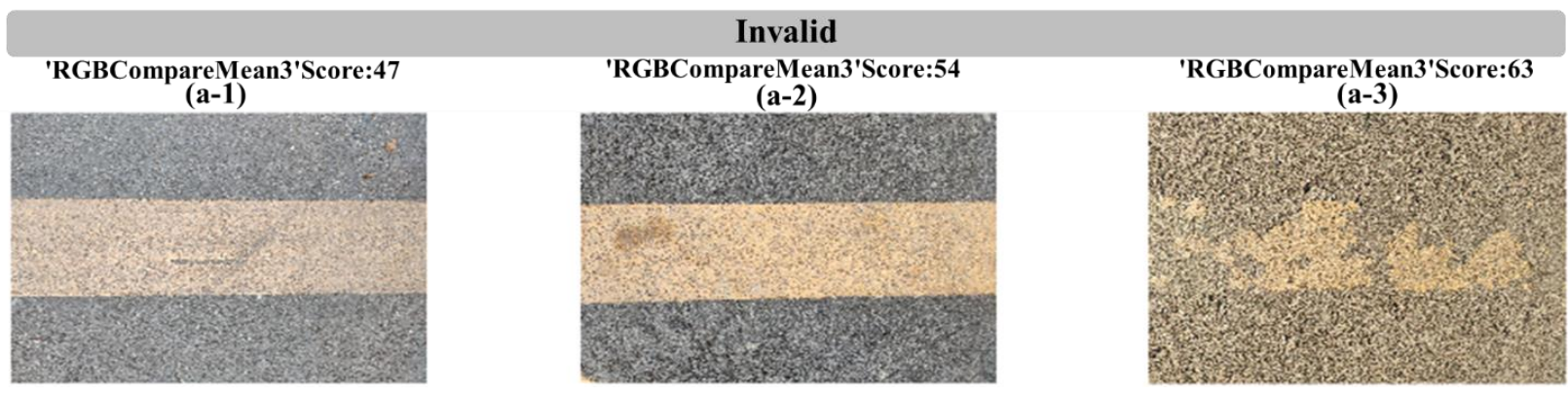

Fig. 2 Index screening based on extreme value samples, Group (a) are the quantification index of color conformity. lane lines with worse consistency get higher scores. Obviously the index does not have legitimacy.

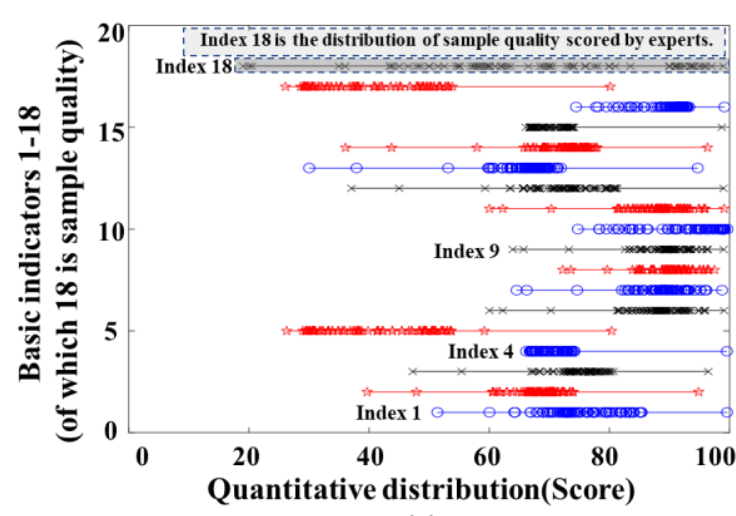

(a)

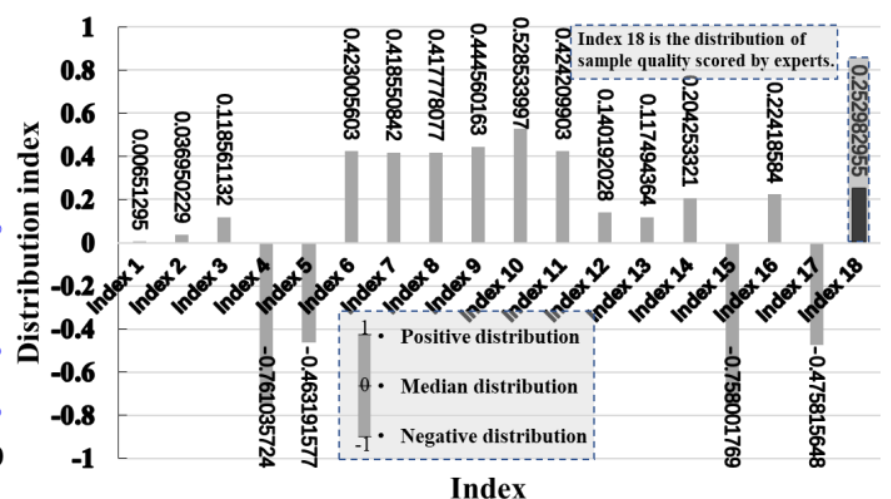

(b)

Fig. 3 The distribution of the quantitative value of the indicator

$$
k=\frac{\operatorname{sum}(\text { compare }(\operatorname{diff}(\text { Data }), \operatorname{diff}(\text { Data } 2)))}{n}
$$

Where $\operatorname{diff(Data)}$ is used to obtain the growth trend of the data, such as formula Error! Reference source not found.. And compare(diff (Data1), diff(Data2)) is used to compare the identity of the two sets of data, such as formula Error! Reference source not found.. $\mathrm{N}$ represents the total number of samples.

$$
\begin{aligned}
& \operatorname{diff}(\text { Data })=\left[s_{1}=0, s_{2}, \ldots, s_{n}\right], s_{i}=\left\{\begin{array}{cc}
1 & \text { if },\left(x_{i+1}-x_{i}\right) \geq 0 \\
-1 & \text { if },\left(x_{i+1}-x_{i}\right)<0
\end{array}(i=1,2,3, \ldots, n-1)\right. \\
& \text { compare (diff (Data1), diff }(\text { Data2 }))=\left[\mathrm{c}_{1}, \mathrm{c}_{2}, \ldots, \mathrm{c}_{n}\right], \mathrm{c}_{i}= \begin{cases}1 & \text { if }, d_{x i} \neq d_{y i} \\
0 & f, d_{x i}=d_{y i}\end{cases} \\
& \text { Among them, } d_{x i} \subset \operatorname{diff}\left(\text { Data1), } d_{y i} \subset \operatorname{diff}(\text { Data2) }\right.
\end{aligned}
$$




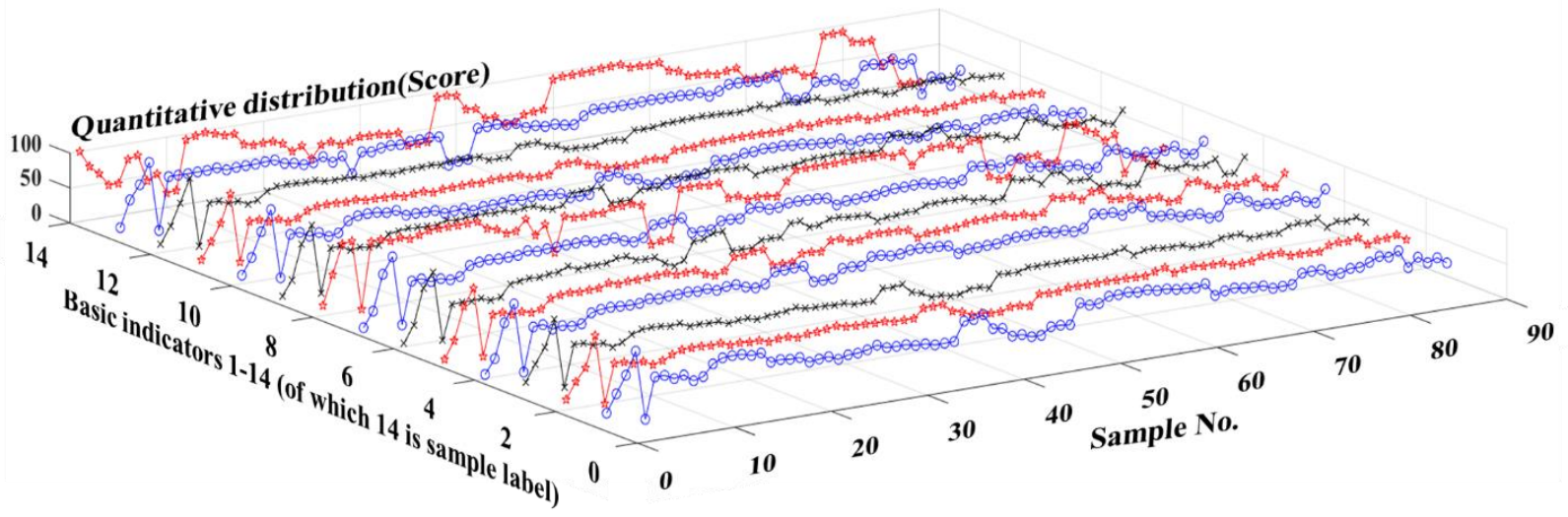

Fig. 4 The quantitative value curve of the indicator

Combined the basic indicators, it obtained the evaluation model of AHP, as shown in Fig. 6. In order to get the evaluation model's parameters, it is necessary to evaluate the relative importance of the model elements in the same dimension[21-22]. In the original construct method of the judgment matrix, when there are many model variables, the consistency check of the judgment matrix is prone to fail. If fail in check, it has to re-evaluation to optimize the matrix. This article proposed a new scoring method to improve the process, which can quickly establish an index system and avoid possible problem in consistency caused by the traditional scoring, the improved process has shown in Fig. 5. And there is example has shown in TABLE A2 \& TABLE A3. The improved expert scoring is mainly based on the importance of the inter-layer matrix shown in TABLE A2. The scoring range of indicator elements from 1 to 9, and its score's meaning include: 1)The higher the score means higher importance; 2)The greater difference between the scores of the elements means higher relative importance; 3)The same score means same importance. After obtaining the order table of importance, according to formula (5), the relative importance index between the elements and the inter-layer element judgment matrix can be further formed.

$$
\left\{\begin{array}{cl}
i_{A B}=\left(A_{\text {score }}-B_{\text {score }}\right)+1 & \text { The importance of } \mathrm{A} \text { relative to } \mathrm{B}\left(\mathrm{A}_{\text {score }} \geq \mathrm{B}_{\text {score }}\right) \\
i_{B A}=1 / i_{A B} & \text { The importance of } \mathrm{B} \text { relative to } \mathrm{A}\left(\mathrm{A}_{\text {score }} \geq \mathrm{B}_{\text {score }}\right)
\end{array}\right.
$$

For example, elements $A_{\text {score }}=9$ and $B_{\text {score }}=1$. According to the formula, the relative importance value of $A$ to $B$ is 9, and the corresponding meaning reference [21] is "indicating that two factors are more important than the other." B relative to A is 1/9, and the corresponding meaning[21] is "compared to two factors, one factor is extremely unimportant than the other." Appendix TABLE A2 \& TABLE A3 has shown the generation of the judgment matrix taking the criterion layer of the AHP model in this paper as an example. The consistency of this example satisfies requirement[21].

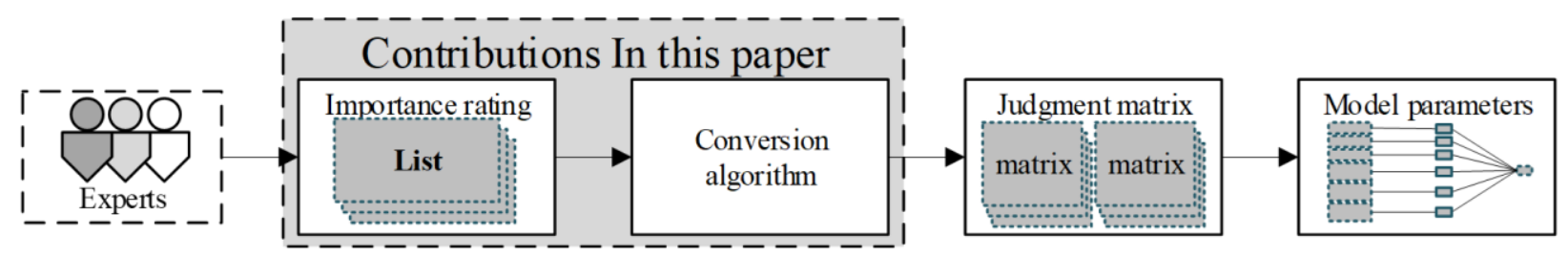

Fig. 5 Establishment of AHP model used in this article

According to the improved scoring method, it adopted the importance scores of seven drivers (in order to keep the data consistent between AHP weight and validation sample data set, the validation data was done by the same personnel, in sector 2.3). For keeping objectivity, taken the rounded mean value of each sample score as the final value. The final weight and its CR (Concordance ratio) based on experts' score has appended to TABLE A1, it can be seen that the model meets the consistency check [21].

\subsection{BP neural network model}


According to existing feature data, with a total of 84 groups of samples, each sample contains 21 quality indicators, the true value of the sample has marked by 7 drivers mentioned above through special software, as shown in Fig. 6(A). For reducing the subjectiveness of experts in scoring, the range was set from 0 to 100 and divided into 5 grade intervals, including very poor (unqualified) 0 60 , poor 60-70, fair 70-80, good 80-90, excellent 90-100. Through this scoring method, it assumed that experts' subjectivity will limit to score fluctuations within the interval. And, Certain threshold $(60 \%$ is used in the following) has use to divide the data into two types, qualified and unqualified, to further reduce the impact of subjectiveness. In addition, the averaging operation to 7 data groups may reduce the subjectivity of the data to a certain extent. The some images and its scores have shown in Fig. 6(B). For BP network training and verification, the data set has randomly divided into two groups, including Train Data(80\%) and Validation Test Data(20\%).

The BP network structure refers to the structural characteristics of the AHP model, as shown in Fig. 6(C), in order to obtain reasonable model parameters , the number of internal neural nodes in intermediate layer network $[A, B, C, D, E, F]$ has set as a variable, and the range of change was $K: 1-100$, the corresponding scale of weights is $K *(21+6)$. In addition, four common transfer functions, including purelin, poslin, logsig, and tansig, have used as candidates of the interlayer's transfer function, their respective activation characteristics have shown in the lower part of Fig. 6(C). Integrating transfer function combination(16 types in total) in four different options and the network nodes' scale that vary from 1 to 100 between layers to forms a parameter space with 1600 different network structures.

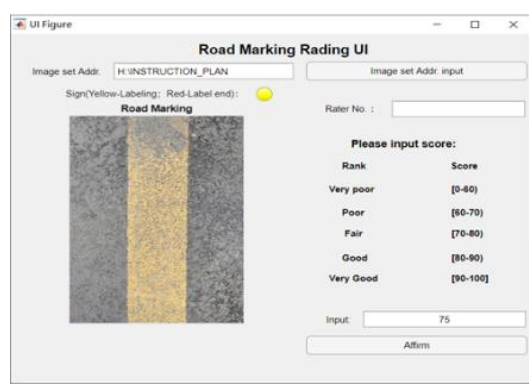

(A)

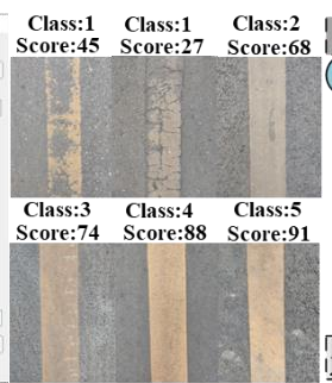

(B)

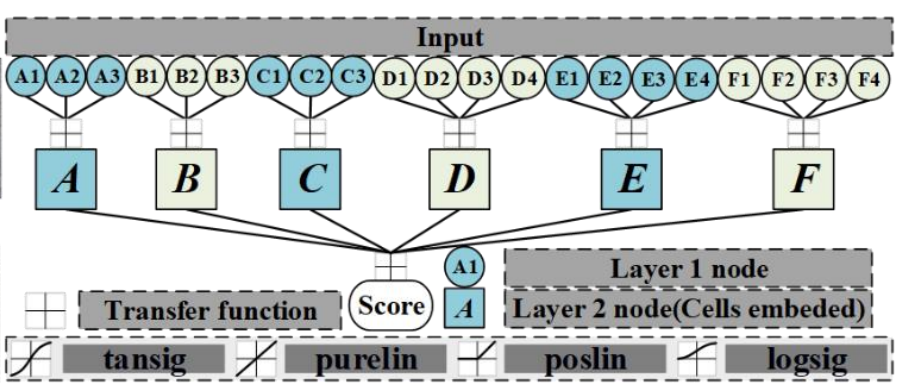

(C)

Fig. 6 (A) the user interface for lane lines scoring;(B) Part of the labelled data in this article;(C) AHP-BP Net has the same basic structure as the AHP model except for the transfer function and the internal network nodes in the middle layer.

Table 1 NP Net Performance Val. Max Err represents the maximum error in the test set; Tol. Max Err is the maximum error under the test of the entire data set; Accuracy (\%), Precision (\%), Recall (\%), are the pass \& fail classification performance (pass threshold $=60$ )

\begin{tabular}{ccccccccc}
\hline \multirow{2}{*}{ Mode } & $\begin{array}{c}\text { layer1 } \\
\text { Transfc }\end{array}$ & $\begin{array}{c}\text { Layer2 } \\
\text { Transfc }\end{array}$ & $\begin{array}{c}\text { Layer1 Cell } \\
\text { Num }\end{array}$ & Val. Max Err & Tol. Max Err & $\begin{array}{c}\text { Accuracy } \\
(\%)\end{array}$ & $\begin{array}{c}\text { Precision } \\
(\%)\end{array}$ & $\begin{array}{c}\text { Recall } \\
(\%)\end{array}$ \\
\hline$[15]$ & - & - & - & - & - & 76.67 & $\mathbf{1 0 0}$ & 50 \\
\hline & tansig & purelin & $\mathbf{8 1}$ & $\mathbf{1 1 . 4 6}$ & $\mathbf{2 2 . 6 2}$ & $\mathbf{1 0 0}$ & $\mathbf{1 0 0}$ & $\mathbf{1 0 0}$ \\
AHP- & purelin & purelin & $\mathbf{1 8}$ & $\mathbf{8 . 0 7 2}$ & $\mathbf{2 5 . 4 9}$ & $\mathbf{9 7 . 5 9}$ & $\mathbf{9 8 . 3 0}$ & $\mathbf{9 8 . 3 0}$ \\
BP & tansig & tansig & 91 & 11.32 & 31.34 & 97.59 & 98.30 & 98.30 \\
Net & poslin & poslin & $\mathbf{1 4}$ & $\mathbf{1 1 . 3 4}$ & $\mathbf{3 6 . 3 9}$ & $\mathbf{9 8 . 7 9}$ & $\mathbf{1 0 0}$ & $\mathbf{9 8 . 3 0}$ \\
& $\ldots$ & $\ldots$ & $\ldots$ & $\ldots$ & $\ldots$ & $\ldots$ & $\ldots$ & $\ldots$ \\
& \multicolumn{2}{c}{ Mean } & $\mathbf{5 8 . 6 8}$ & $\mathbf{1 0 . 5 8}$ & $\mathbf{2 5 . 6 9}$ & $\mathbf{9 7 . 2 1}$ & $\mathbf{9 8 . 6 1}$ & $\mathbf{9 7 . 4 5}$ \\
\hline
\end{tabular}

The networks have trained by randomly dividing $80 \%$ of the training data, and tested by the remaining $20 \%$ of the data, as the result, the maximum error of each network in test sample set employed to further screen different networks. These networks and corresponding performances, have shown in Table 1 , included 16 kinds of networks with different transfer functions and optimal 
network nodes. Performance indicators in Table 1 mainly included the maximum error of the test set, the maximum error of the full sample set, and the classification(Pass or Fail(0\&1)performance indicators (Accuracy (\%), Precision (\%), Recall (\%), and divided the qualified lane line by $60 \%$ threshold[15]).

Through Table 1, following results can be summed up:

1) When AHP-BP net was set as tansig-poslin-81, compared with other configuration, it has better performance in $0 \& 1$ classification, and the other configurations performed well in this aspect without significant difference.

2) In verification set and full set test, the performance of tansig-poslin-18 is higher than the average, but weak, its classification performance is average.

3)For the network parameter scale, the poslin-poslin-14 network has the best set, compared to the tansig-poslin-18 and the whole mode compared with the optimal performance network(tansigposlin-81) and average reduced to $17.28 \%$ and $23.89 \%$, respectively.

The average network models equipped other 12 settings are omitted here. Based on the above results, it adopt the network tansig-poslin-81 performed best performance in $0 \& 1$ classification and the lightweight network poslin-poslin-14 as the optimal model.

\section{Experimental results and Discussion}

Table 2 Evaluation Model Performance. Val. Max Err represents the maximum error in the test set; Tol. Max Err is the maximum error under the test of the entire data set; Accuracy (\%), Precision (\%), Recall (\%), are the qualified \& unqualified classification performance (pass threshold $=60$ )

\begin{tabular}{cccccccc}
\hline Mode & $\begin{array}{c}\text { True } \\
\text { positive }\end{array}$ & $\begin{array}{c}\text { False } \\
\text { positive }\end{array}$ & $\begin{array}{c}\text { False } \\
\text { negative }\end{array}$ & $\begin{array}{c}\text { True } \\
\text { negative }\end{array}$ & $\begin{array}{c}\text { Accuracy } \\
(\%)\end{array}$ & $\begin{array}{c}\text { Precision } \\
(\%)\end{array}$ & $\begin{array}{c}\text { Recall } \\
(\%)\end{array}$ \\
\hline $\begin{array}{c}\text { [15] } \\
\text { tansig- }\end{array}$ & $\mathbf{7}$ & 0 & $\mathbf{7}$ & 16 & 76.67 & 100 & 50 \\
$\begin{array}{c}\text { purelin-81 } \\
\text { poslin-poslin- }\end{array}$ & $\mathbf{5 9}$ & $\mathbf{0}$ & $\mathbf{0}$ & $\mathbf{2 4}$ & $\mathbf{1 0 0}$ & $\mathbf{1 0 0}$ & $\mathbf{1 0 0}$ \\
$\mathbf{1 4}$ & $\mathbf{5 8}$ & $\mathbf{0}$ & $\mathbf{1}$ & $\mathbf{2 4}$ & $\mathbf{9 8 . 7 9 5 1 8}$ & $\mathbf{1 0 0}$ & $\mathbf{9 8 . 3 0 5 0 8}$ \\
Mean & 57.5 & 0.8125 & $\mathbf{1 . 5}$ & $\mathbf{2 3 . 1 8 7 5}$ & 97.21385 & 98.61715 & 97.45763 \\
\hline AHP & $\mathbf{5 9}$ & $\mathbf{0}$ & $\mathbf{1 8}$ & $\mathbf{6}$ & $\mathbf{7 8 . 3 1 3 2 5}$ & $\mathbf{1 0 0}$ & $\mathbf{7 6 . 6 2 3 3 8}$ \\
\hline
\end{tabular}

The main performance of the comprehensive evaluation models obtained in this paper have shown in Table 2. Comparing the best performance mode and the smallest parameter scale mode in the AHP-BP network model with the classic AHP evaluation method and the method in literature [15], the following conclusions are drawn:

1. The model adopted in this paper expands the characteristic dimensions of the existing lane line quality recognition classification, so that the lane line quality evaluation meets the actual standard, and the evaluation model is feasible.

2、 Using parameter search method to construct the AHP-BP network has better performance than AHP model, including an optimal performance network and a lightweight network.

3、 Optimized the AHP-BP method(BP-Mode-1- tansig-purelin-81) performed excellent classification in given database. Compared with the AHP model, Accuracy increased by $21.68 \%$, and Recall increased by $23.37 \%$.Lightweight model(BP-Mode-1- poslin-poslin-14) has $82 \%$ fewer parameters than the optimal network (BP-Mode-1- tansig-purelin-81). Compared with the AHP model, the Accuracy is increased by $20 \%$, and the Recall is increased by $18 \%$. 
4、 The AHP model and the two AHP-BP Network model obtained in this paper have used in the lane line qualification assessment, and their performance is greatly improved compared with the method similar literature reported.

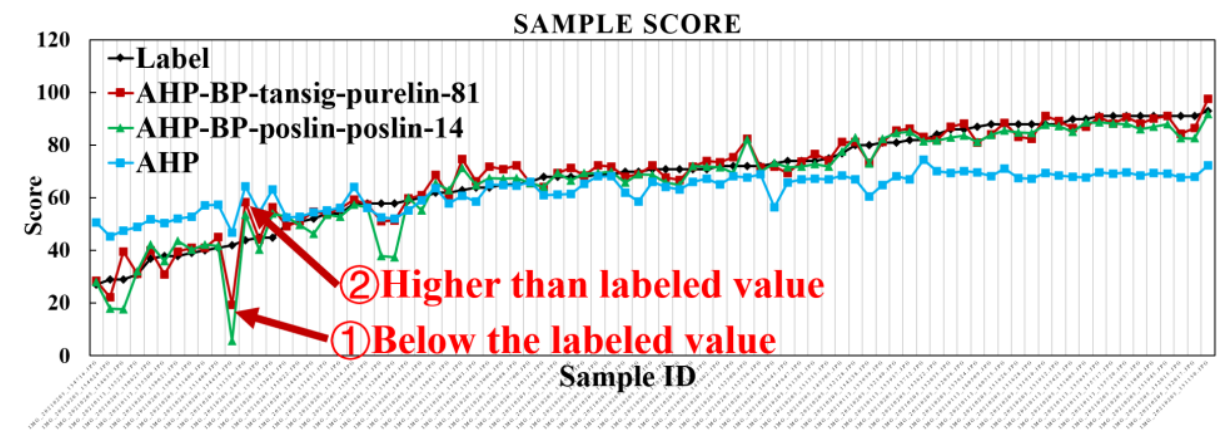

Fig. 7 The comparison curve between the predicted value of the model and the marked value. The modified figure shows that the model proposed in this article has better fitting performance than the AHP model.

In addition, this paper compared the model prediction values of all samples with the labeled values. As shown in the Fig. 7, it can be clearly seen that the optimal model and the lightweight model have a better fitting effect than the AHP model. However, the two AHP-BP models have large prediction errors on the two highlight points indicated in the Fig. 8 and Fig. 9. By obtaining the samples and data corresponding to the two points, as shown in the Fig. 8, we find that the preprocessing of the samples corresponding to the first point has actually been identified as a large number of lane marking blockiness (15 color blocks), and the corresponding feature quantification is compared with The number of blocks is inversely proportional, but in the manual evaluation, the lane line is actually not as negative as the program judgment, so the model prediction value is lower than the marked value. For the second point corresponding sample, Fig. 9 , through analysis, it is found that the lane line defacement defect was correctly identified, but the labeler was able to identify the defect and give a negative score, which resulted in a large error between the label value and the predicted value. (In order to confirm the rationality of the explanation, another similar sample and corresponding value was added to the figure to confirm it.) It can be seen that the preprocessing procedures and defect feature quantification methods used in the model in this paper can be further optimized.
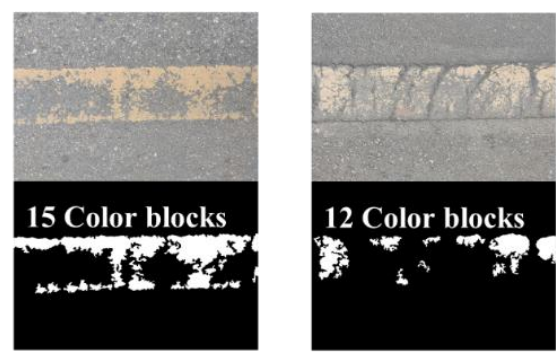

\begin{tabular}{ccc}
\hline Model & 1-Score & 2-Score \\
\hline Model-81 & $\mathbf{1 9 . 3 7 6 2}$ & 22.3585 \\
Model-14 & $\mathbf{5 . 6 0 4 2}$ & 17.9138 \\
AHP & $\mathbf{4 6 . 8 2 2 5}$ & 45 \\
Label & $\mathbf{4 2}$ & 29 \\
\hline
\end{tabular}

Fig. 8 Sample-left (1-Score) is the sample corresponding to the first point in the comparison label, and sample-right (1-

Score2) is a similar sample; this example shows that the feature quantization method is unreasonable and leads to
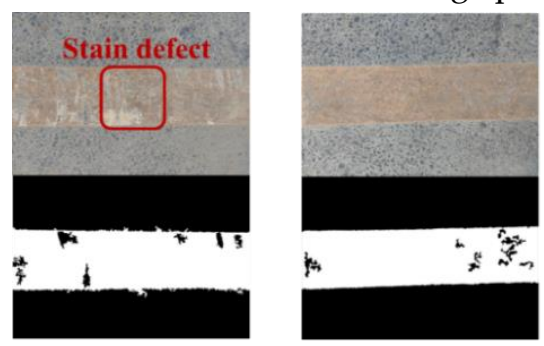

\begin{tabular}{ccc}
\hline Model & 1-Score & 2-Score \\
\hline Model-81 & $\mathbf{5 8 . 4 5 7 3}$ & 59.2126 \\
Model-14 & $\mathbf{5 3 . 4 8 2 4}$ & 57.4226 \\
AHP & $\mathbf{6 4 . 2 7 9 7}$ & 64.126 \\
Label & $\mathbf{4 4}$ & 58 \\
\hline
\end{tabular}


Fig. 9 Sample-left (1-Score) is the sample corresponding to the second point in the comparison annotation, and sample-right (1-Score2) is a similar sample; this example shows that the preprocessing algorithm identifies color pollution defects and causes large prediction errors

\section{Conclusion}

Based on the machine vision method, this paper proposes an algorithm that extracts multiple lane line quality feature dimensions, which provides necessary conditions for the timely and good lane line maintenance evaluation. Based on the feature extraction algorithm, a multi-dimensional lane line quality evaluation method is constructed. And employed the lane line quality data set scored by experts, it researched the best AHP-BP model and the lightweight model through the network structure search method within a certain range. Through verification and comparison, the multi-dimensional feature extraction algorithm and optimization evaluation model proposed in this paper show effectiveness in lane line quality classification.

The method proposed in this paper is based on local image sampling, and the application of large-scale road quality monitoring needs to be assisted with corresponding special equipment and integration strategies. In addition, because of the inherent nature of classic machine vision method, strong sunlight and shadows may have certain interference with color quality quantification, and follow-up work will devoted to solving those pre-processing defects. Due to the limitations of expert scoring method for training and verification, work will be carried out on how to build an objective, stable and reliable data set from now on. The obtained neural network model can be further studied and optimized in terms of computing speed and computing resource requirements.

\section{Appendix A}

TABLE A1 Analytic Hierarchy Model Index and its corresponding weight

\begin{tabular}{|c|c|c|c|c|c|}
\hline CR & $\begin{array}{l}\text { Criterion } \\
\text { Layer }\end{array}$ & Weight & $\mathrm{CR}$ & Basic indicators & Weight \\
\hline \multirow{15}{*}{$\begin{array}{l}0.0051 \\
057\end{array}$} & \multirow{3}{*}{$\begin{array}{l}\text { Color } \\
\text { ntity }\end{array}$} & \multirow{3}{*}{0.175726} & \multirow{3}{*}{0.024065} & A1 RGB-channel B standard deviation & 0.09739 \\
\hline & & & & A2 LAB- channel L standard deviation & 0.333069 \\
\hline & & & & A3 HSV- channel H standard deviation & 0.569541 \\
\hline & \multirow{3}{*}{$\begin{array}{l}\text { B Color } \\
\text { conformity }\end{array}$} & \multirow{3}{*}{0.175726} & \multirow{3}{*}{$2.13 \mathrm{E}-15$} & $\begin{array}{l}\text { B1 Average difference between RGB-R } \\
\text { channel and standard value }\end{array}$ & 0.428571 \\
\hline & & & & $\begin{array}{l}\text { B2 Standard deviation of RGB-R channel } \\
\text { to standard value }\end{array}$ & 0.428571 \\
\hline & & & & $\begin{array}{l}\text { B3 Standard deviation of HSV-H channel } \\
\text { to standard value }\end{array}$ & 0.142857 \\
\hline & \multirow{3}{*}{$\begin{array}{l}\text { C Shape } \\
\text { conformity }\end{array}$} & \multirow{3}{*}{0.097213} & \multirow{3}{*}{0.016938} & C1 Effective area ratio & 0.558425 \\
\hline & & & & C2 Blocking factor & 0.121957 \\
\hline & & & & C3 Fracture coefficient & 0.319618 \\
\hline & \multirow{4}{*}{$\begin{array}{l}\text { D Width } \\
\text { compliance }\end{array}$} & \multirow{4}{*}{0.175726} & \multirow{4}{*}{0.0038421} & D1 Minimum width & 0.227044 \\
\hline & & & & D2 Maximum width & 0.122324 \\
\hline & & & & D3 Mean width & 0.423587 \\
\hline & & & & D4 Fracture coefficient & 0.227044 \\
\hline & \multirow{2}{*}{$\begin{array}{l}\text { E Edge } \\
\text { continuity }\end{array}$} & \multirow{2}{*}{0.060246} & \multirow{2}{*}{0.0039841} & $\begin{array}{l}\text { E1 Width standard deviation based on the } \\
\text { width of the mode }\end{array}$ & 0.227044 \\
\hline & & & & $\begin{array}{l}\text { E2 Standard deviation of width based on } \\
\text { maximum value }\end{array}$ & 0.227044 \\
\hline
\end{tabular}


E3 Width standard deviation based on the mean

0.423587

E4 Fracture coefficient

0.122324

F1 Mean difference based on mean gray 0.350913

F2 Mode difference based on mean gray

F Contrast

level

0.350913

with road $0.315362 \quad 0.0039386$

color

F3 Mean difference based on Lab-B

channel

0.109114

F4 Mode difference based on Lab-B

channel

0.18906

TABLE A2 Importance ranking reference table and Example

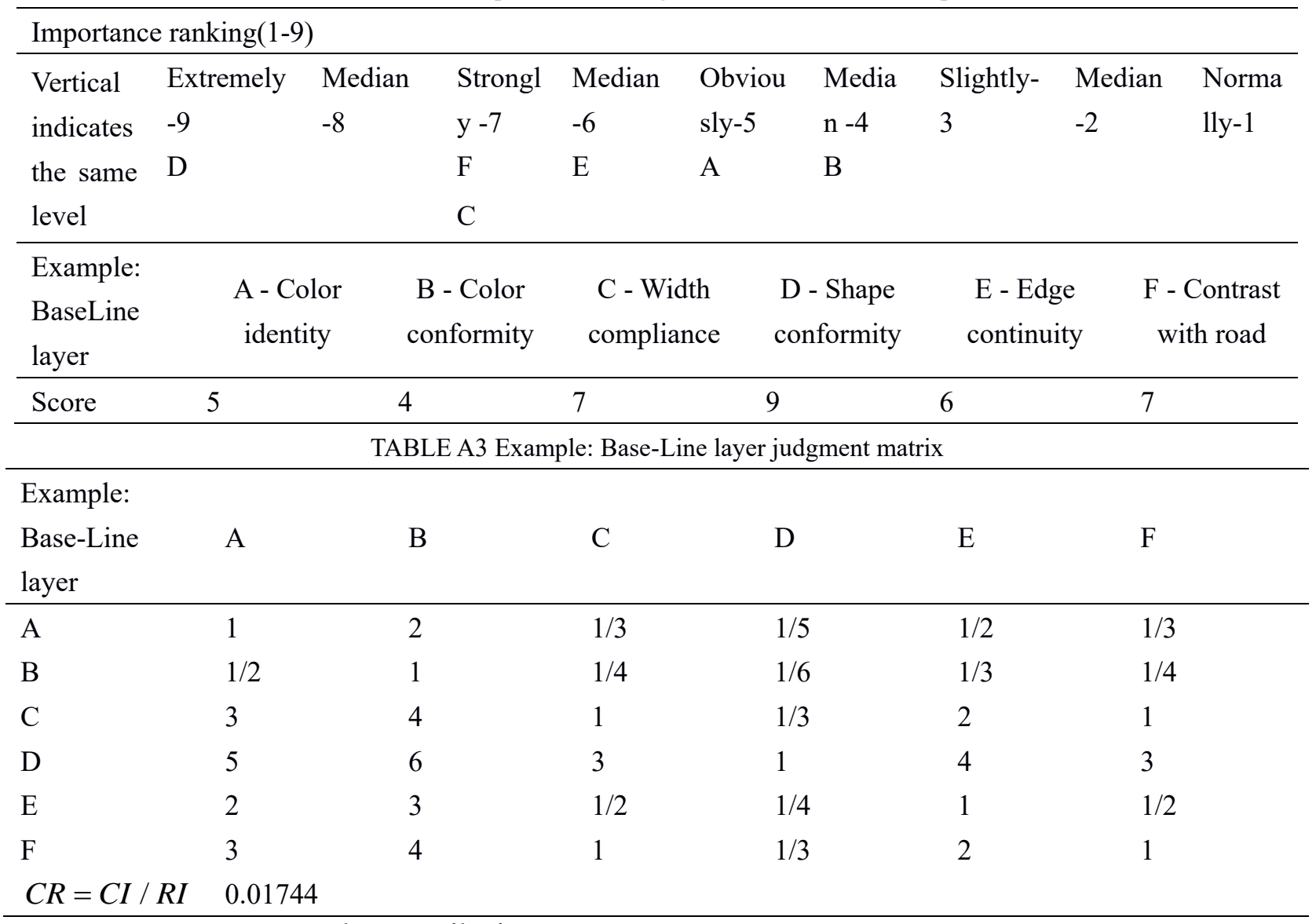

\section{Author Contributions:}

H.W.: Conceptualization, Methodology, Software, Original Draft; P.Y.D.: Conceptualization, Methodology,Original Draft, Review \& Editing; S.L.S.: Review \& Editing; G.Y.T.: Review \& Editing; S.G.L.: Software; N.Y.L.: Formal analysis; C.L.K.: Formal analysis; J.B.Z.: Software; Y.Q.P.: Review; J.J.L.: Review \& Editing;All authors have read and agreed to the published version of the manuscript.

Funding: This article was generously funded by the following projects of the Key R\&D Projects of Science \& Technology Department of Sichuan Province, including Project 2021YFG0070, 2020YFG0325, 2019 YFG0528 and 2019YFG0367.

Conflicts of Interest: The authors declare no conflict of interest.

\section{References}


1. Burningham, S.; Stankevich, N. Why Road Maintenance is Important and How to Get it Done. Transport Note 2005, 4, $1-10$.

2. Hillel, A. B.; Lerner, R.; Levi D, Raz, G. Recent Progress in Road and Lane Detection: a Survey. Machine Vision and Applications 2014, 25, 727-745.

3. Storsaeter, A.D.; Pitera, K.; Mccormack, E.D. The Automated Driver as a New Road User. Transport Reviews 2020, 1-23, DOI: 10.1080/01441647.2020.1861124.

4. Pasetto, M.; Barbati, S.D. Experimental Investigation on Road Marking Distress Evolution: Beyond Testing. Quality Assurance and Maintenance Improvement, Advanced Materials Research, 2013, 723, 846-853.

5. Supervision Jiangsu-Provincial-Bureau-of-Quality-and-Technical. Ordinary National and Provincial Highways Signs and Markings Maintenance Standard: DB 32/T 3493-2019, 2019.

6. Wickramarathne, T.; Garg, V.; Bauer P. On the Use of 3-d Accelerometers for Road Quality Assessment. 2018 IEEE 87th Vehicular Technology Conference (VTC Spring) 2018, 1-5.

7. Sattar, S.; Li, S.; Chapman, M. Road Surface Monitoring Using Smartphone Sensors: a Review. Sensors 2018, 18, 3845 .

8. Zhang, D.; Li, Q.; Chen, Y.; He, L.; Zhang, B. An Efficient and Reliable Coarse-to-fine Approach for Asphalt Pavement Crack Detection. Image and Vision Computing 2017, 57, 130-146.

9. Abou.; Chacra, D.B.; Zelek, J.S. Fully Automated Road Defect Detection Using Street View Images. In 2017 14th Conference on Computer and Robot Vision (CRV) 2017, 353-360.

10. Shi, Y.; Cui, L.; Qi, Z.; Meng, F.; Chen, Z. Automatic Road Crack Detection Using Random Structured Forests. In IEEE Transactions on Intelligent Transportation Systems 2016, 17, 3434-3445

11. Wen, C.; Sun, X.; Li, J.; et al. A Deep Learning Framework for Road Marking Extraction, Classification and Completion From Mobile Laser Scanning Point Clouds, Isprs Journal of Photogrammetry and Remote Sensing 2019, $147,178-192$

12. Ma, L.; Li, Y.; Li, J.; Yu, Y.; Junior, J. M.; Gonçalves, W. N.; Chapman, M. A. Capsule-based Networks for Road Marking Extraction and Classification From Mobile Lidar Point Clouds. In IEEE Transactions on Intelligent Transportation Systems 2020, 22,4,1981-1995

13. Lee, S.; Kim, J.; Shin yoon J.; Shin, S.; Bailo, O.; Kim, N.; So Kweon, I. VPGNet: Vanishing Point Guided Network for Lane and Road Marking Detection and Recognition. In Proceedings of the IEEE International Conference on Computer Vision 2017: 1947-1955.

14. Burrow, M.; Evdorides, H.; Snaith, M. Road Marking Assessment Using Digital Image Analysis, Proceedings of the Institution of Civil Engineers-transport 2000: 107-112.

15. Lin, K.; Wu, T.; Wang, Y. An Innovative Road Marking Quality Assessment Mechanism Using Computer Vision, Advances in Mechanical Engineering 2016, 8.

16. Committee CNSM, Road Traffic Signs and Markings: GBT 5768.3-2009.

17. Committee CNSM, Specification and Test Method for Road Traffic Markings: GBT 16311-2009, 2010.

18. Standardization EUROPEAN-COMMITTEE-FOR. Road marking materials - Road marking performance for road users and test methods: ICS 93.080.30, 2018. 
19. Kavdir. İ.; Guyer; D. Evaluation of Different Pattern Recognition Techniques for Apple Sorting. Biosystems Engineering 2008, 99, 211-219.

20. Çetin, N.; Karaman, K.; Beyzi, E. Comparative Evaluation of Some Quality Characteristics of Sunflower Oilseeds (helianthus Annuus L.) Through Machine Learning Classifiers. Food Analytical Methods 2021, 1-16.

21. Saaty, T.L. How to Make a Decision: the Analytic Hierarchy Process. European Journal of Operational Research 1990, 48, 9-26.

22. Saaty, R.W. The Analytic Hierarchy Process — what It Is and How It Is Used. Mathematical Modelling 1987, 9, 161176.

23. Al-Harbi, K.M.A. Application of the Ahp in Project Management, International Journal of Project Management 2001. 19, 19-27.

24. Gupta, A.; Singh, R.K.; Mangla, S.K. Evaluation of Logistics Providers for Sustainable Service Quality: Analytics Based Decision Making Framework. Annals of Operations Research(2021), 1-48.

25. Li, Y.; Wei, M.; Liu, L.; Yu, B.; Dong, Z.; Xue, Q. Evaluation of the Effectiveness of Voc-contaminated Soil Preparation Based on Ahp-critic-topsis Model. Chemosphere 2021, 271, 129571.

26. Xie, Z.; Su, Z. Evaluation of College English Classroom Teaching Quality Dependent on Triangular Fuzzy Number. The International Journal of Electrical Engineering \& Education 2021, dio: 10.1177/00207209211002075.

27. Wenwen, L. Modeling and Simulation of Teaching Quality in Colleges Based on Bp Neural Network and Training Function. Journal of Intelligent \& Fuzzy Systems 2019, 37, 6349-6361.

28. Chen, J.; Huang, S. Evaluation Model of Green Supply Chain Cooperation Credit Based on Bp Neural Network. Neural Computing and Applications 2021, 33, 1007-1015.

29. Dai, W.; Hu, P. Application of Bp Neural Network in the Analytic Hierarchy Process of Person-post Evaluation Model. The Journal of Supercomputing 2020, 76, 897-914.

30. Schwarz, M.W.; Cowan, W.B.; Beatty, J.C. An Experimental Comparison of Rgb, Yiq, Lab, Hsv, and Opponent Color Models. Acm Transactions on Graphics (tog) 1987, 6, 123-158.

31. Otsu, N. A Threshold Selection Method From Gray-level Histograms. In IEEE Transactions on Systems, Man, and Cybernetics 1979, 9, 1, 62-66.

32. Weiss, B. Fast Median. Bilateral Filtering, ACM Siggraph 2006 Papers 2006, 519-526. 